\title{
Two-photon imaging induces brain heating and calcium microdomain hyper- activity in cortical astrocytes
}

[70/135 characters]

Elke Schmidt ${ }^{\mathrm{a}, \mathrm{b}}$ and Martin Oheim, ${ }^{\mathrm{a}, \mathrm{b}, *}$

${ }^{a}$ Centre National de la Recherche Scientifique, CNRS, UMR8118, Brain Physiology Laboratory, 45 rue des Saints Pères, Paris, F-75006 France;

${ }^{\mathrm{b}}$ Fédération de Neurosciences, CNRS, FR3636, Faculté des Sciences Fondamentales et Biomédicales, Université Paris Descartes, PRES Sorbonne Paris Cité, 45 rue des Saints Pères, Paris, F-75006 France;

*ORCID id: orcid.org/0000-0001-8139-167X

corresponding author

Phone : $\quad++33142864222$ (office), -21 (lab)

E-mail : $\quad$ martin.oheim@parisdescartes.fr

CLASSIFICATION: $\quad$ Major category: Biological Sciences

Minor categories: Neuroscience / Physiology

AUTHOR CONTRIBUTIONS: ES and MO designed and performed research, ES analyzed the data, MO wrote the paper.

CONFLICT OF INTEREST STATEMENT: The authors declare no conflicting interest. 
ABSTRACT Unraveling how neural networks process and represent sensory information and how this cellular dynamics instructs behavioral output is a main goal in current neuroscience. Two-photon activation of optogenetic actuators and fluorescence calcium $\left(\mathrm{Ca}^{2+}\right)$ imaging with genetically encoded $\mathrm{Ca}^{2+}$ indicators allow, respectively, the all-optical stimulation and readout of activity from genetically identified cell populations. However, these techniques expose the brain to high near-infrared light doses raising the concern of light-induced adverse effects on the biological phenomena being studied. Combing $\mathrm{Ca}^{2+}$ imaging of GCaMP6f-expressing cortical astrocytes as a sensitive readout for photodamage and an unbiased machine-based event detection, we demonstrate the subtle build-up of aberrant microdomain $\mathrm{Ca}^{2+}$ signals in fine astroglial processes. Illumination conditions routinely being used in biological twophoton microscopy (920-nm excitation, 100-fs regime, ten $\mathrm{mW}$ average power) increased the frequency of microdomain $\mathrm{Ca}^{2+}$ events, but left their amplitude, area and duration rather unchanged. This increase in local $\mathrm{Ca}^{2+}$ activity was followed by $\mathrm{Ca}^{2+}$ transients in the otherwise silent soma. $\mathrm{Ca}^{2+}$ hyperactivity occurred without overt morphological damage. Surprisingly, at the same average power, continuous-wave 920-nm illumination was as damaging as fs pulses, indicating a linear, heating-mediated (rather than a highly non-linear) damage mechanism. In an astrocyte-specific $\mathrm{IP}_{3}$-receptor knock-out mouse $\left(\mathrm{IP}_{3} \mathrm{R} 2-\mathrm{KO}\right)$, Near-infrared light-induced $\mathrm{Ca}^{2+}$ microdomains signals persisted in the small processes, underpinning their resemblance to physiological $\mathrm{IP}_{3} \mathrm{R} 2$-independent $\mathrm{Ca}^{2+}$ signals, while somatic activity was abolished. Contrary to what has generally been believed in the field, shorter pulses and lower average power are advantageous to alleviate photodamage and allow for longer useful recording windows. [250/250 words]

KEYWORDS: Glia, calcium microdomains, non-linear imaging, GCaMP, two-photon, photodamage, temperature 


\section{SIGNIFICANCE STATEMENT}

Imaging the fine structure and function of the brain has become possible with two-photon microscopy that uses ultrashort-pulsed infrared laser light for better tissue penetration. The high peak energy of these light pulses has raised concerns about photodamage resulting from multi-photon processes. Here, we show that the time-averaged rather than the peak laser power matters. At wavelengths and with laser powers now commonly used in neuroscience brain damage occurs as a consequence of direct infrared light absorption, i.e., heating. To counteract brain heating we explore a strategy that uses even shorter, more energetic pulses but a lower time-averaged laser power to produce the same image quality while making twophoton microscopy less invasive. [112/120 words]

\section{lbody}

\section{INTRODUCTION}

Two-photon (2P) excitation fluorescence microscopy (1) is the method of choice for imaging structural and functional dynamics in the intact brain (2-4). Addressing the role of genetically identified cell populations in a circuit-specific manner has become possible through the advent and continuous improvement of genetically encoded calcium $\left(\mathrm{Ca}^{2+}\right)$ indicators (GECIs) (5-7). Considerable efforts are being made to enhance the speed, spatial resolution and field-of-view of $2 \mathrm{P} \mathrm{Ca}^{2+}$ imaging, but for a given GECI the user has to trade off gains in one of these parameters against losses in others, unless the number of excitation spots and/or the average laser power $\bar{P}$ per spot are increased.

2P fluorescence excitation requires high instantaneous peak intensities, which has prompted concerns about non-linear photobleaching (8) and photodamage resulting from excited-state absorption restricted to the high-intensity focus $(9,10)$. Several studies have established a steep intensity threshold for this non-linear damage. The occurrence of aberrant, 
light-induced $\mathrm{Ca}^{2+}$ signals provides a sensitive readout for assessing damage (9-11). Average laser powers of the order of tens of $\mathrm{mW}$ per excitation spot are generally being considered safe (11), and up to $250 \mathrm{~mW}$ have been reported in multi-spot excitation schemes (12). How different pulse parameters (pulse length, inter-pulse interval, peak power) affect this damage threshold has remained controversial.

In the current work, we demonstrate an unexpected photodamage that affects physiological astrocytic $\mathrm{Ca}^{2+}$ signals, occurs upon 920-nm excitation at low $\mathrm{mW}$ powers, and involves a linear power dependence. Unbiased automated analysis of $\mathrm{Ca}^{2+}$ signals revealed a subtle but measurable build-up of spontaneous, microdomain $\mathrm{Ca}^{2+}$ activity in the fine processes of GCaMP6f-expressing cortical astrocytes under conditions routinely used for $2 \mathrm{P}$ brain imaging. Near-infrared (NIR) illumination increased the frequency whilst only slightly affecting the amplitude and cellular volume fraction encompassed by these $\mathrm{Ca}^{2+}$ transients. This frequency increase occurred without overt morphological damage. The build-up of aberrant microdomain $\mathrm{Ca}^{2+}$ signals was followed by $\mathrm{Ca}^{2+}$ activity in the otherwise silent soma. Pausing acquisitions reduced light-induced $\mathrm{Ca}^{2+}$ hyperactivity, indicating its dependence on the total light dose rather the timing with respect to the first image. Longer, less energetic pulses and higher average power (resulting in the same signal) were equally harmful. As pulse stretching did not decrease the photoinduced $\mathrm{Ca}^{2+}$ signals, as would have been expected for a non-linear damage mechanism, we postulated a linear (heating-mediated) damage process. Consistent with a one-photon absorption process, continuous-wave 920-nm illumination was equally damaging to small astrocyte processes as were fs pulses.

Our experiments demonstrate that one- rather than two-photon NIR-absorption is limiting in the now popular wavelength band above $900 \mathrm{~nm}$. This has important consequences for imaging fluorescent proteins and functional indicators as well as for studies using optical 
stimulation for optogenetic activation. Thus, a careful optimization of the pulse length whilst reducing the average laser power better preserves the biology under study. (Intro 464 words)

\section{RESULTS}

\section{'Spontaneous' astrocyte $\mathrm{Ca}^{2+}$ signals increase during two-photon recordings}

In the barrel cortex of adult mice (P33-P46) sparsely expressing GCaMP6f (13) in a subset of astrocytes (Fig. 1A and Fig. S1), 2-photon imaging at $920 \mathrm{~nm}$ revealed a plethora of spontaneous, asynchronous and spatially confined $\mathrm{Ca}^{2+}$ signals (Fig. 1 and Supplementary Movie S1). These microdomain $\mathrm{Ca}^{2+}$ signals occurred in the absence of neuronal stimulation. They were astrocyte-intrinsic because they persisted when blocking neuronal action potentials with TTX $(1 \mu \mathrm{M})$ and they were also observed in pure astrocyte culture (data not shown).

We acquired time-lapse image series (at $0.5 \mathrm{~Hz}$ ) from a single equatorial plane encompassing the soma at diffraction-limited spatial resolution $(146 \mathrm{~nm} / \mathrm{px})$. Regions of elevated $\mathrm{Ca}^{2+}($ Fig $1 A$, bottom $)$ and their corresponding $\mathrm{Ca}^{2+}$ transient waveforms (Fig. 1B, top) were extracted by an unbiased machine-based detection procedure, see Fig. S2 and Online methods. The 'summed activity' trace (showing the cumulative activity in the processes) was dominated by a large number of small $\mathrm{Ca}^{2+}$ transients and a few large events and was otherwise flat, as expected for a predominantly localized, asynchronous $\mathrm{Ca}^{2+}$ activity. Soma showed little, if any, spontaneous activity during the first $4 \mathrm{~min}$ of recording. $\mathrm{Ca}^{2+}$ signals were rare $(11.25 \pm 1.76$ events/min $)$, short-lived $(1.90 \pm 0.10 \mathrm{~s}$ duration $)$, of small amplitude $\left(\Delta F / F_{0}=3.02 \pm 0.12\right)$ and they were confined to tiny sub-regions of individual astrocyte branches (mean area, $9.26 \pm 0.52 \mu \mathrm{m}^{2}$, vs. $3969 \pm 302 \mu \mathrm{m}^{2}$ total astrocyte area, i.e., $\sim 0.2 \%$ of the surface area; median \pm SEM for 8 cells during 4 min recording). Our results are similar to those reported by others (14-17). 
Raster plots on which we ordered active regions according to their distance-to-soma showed $\mathrm{Ca}^{2+}$ transients throughout the neuropil with no obvious localization preference. When we extended the recording duration beyond the initial 4 min, we recognized a first subtle and then increasingly pronounced $\mathrm{Ca}^{2+}$ hyperactivity in the fine processes, Fig. 2A, top. This hyperactivity is easily detected as a rise of the 'summed activity' trace that measures the cumulative activity within the processes (Fig. 2A, bottom, grey trace). Somatic $\mathrm{Ca}^{2+}$ hyperactivity was observed secondary to the photodamage triggered in the cell periphery, (Fig. $2 B$, bottom, red trace). With increasing recording time, astrocytic microdomain $\mathrm{Ca}^{2+}$ signals occurred repeatedly within the same subcellular domains, Fig. 2C. Microdomain events remained initially relatively stereotyped in terms of their area, peak amplitude and duration but their frequency almost doubled $(\times 1.87)$ when comparing p1 (0-4 min) and p2 (4-8 min). Following 16 min of continuous recording, microdomain $\mathrm{Ca}^{2+}$ transients in the processes tripled in frequency and had a $40 \%$-increased $\mathrm{d} F / F_{0}$ amplitude, compared to the beginning (p1), Fig. 2D. Other parameters remained unaltered, indicating that photodamage merely triggered more events. We conclude that both event frequency $(\times 2.94)$ and summed activity $(\times 7.5)$ of the processes are bona fide markers for light-induced microdomain $\mathrm{Ca}^{2+}$ hyperactivity. On the macroscopic level, longer recordings produced a net increase in somatic $\mathrm{Ca}^{2+}$ activity $\left(\times 14.5\right.$, p3 vs. p1), Fig. $3 E$ and Supplementary Movie S2. Aberrant $\mathrm{Ca}^{2+}$ signals occurred in the absence of visible morphological damage, and mice expressing EGFP rather than GCaMP6f in an astrocyte-specific manner showed no obvious photoactivation (data not shown). $\mathrm{Ca}^{2+}$ hyperactivity was observed in astrocytes expressing GCaMP3 (18), a GECI displaying a higher basal fluorescence but reduced $\Delta F / F_{0}$ amplitude (19), excluding GCaMP6f-specific effects (data not shown).

The $\mathrm{Ca}^{2+}$ signal obtained when lumping together the entire neuropil to one single ROI mirrored the evolution with time of the summed activity of all individual detected events in 
the cell periphery (Fig.S3). Thus, (i), although highly branched and segregated into a tortious and diffusionally distant volume, the fine processes behave as a single compartment as far as damage is concerned, and, (ii), the soma behaves differently. The simple 'center-vs-surround' analysis hence provides a facile readout of aberrant light-induced $\mathrm{Ca}^{2+}$ activity in the soma and processes, respectively.

Taken together, our results demonstrate the vulnerability of small astrocyte processes to the exposure with pulsed near-infrared (NIR) light. Imaging conditions routinely used for biological 2P microscopy trigger astroglial $\mathrm{Ca}^{2+}$ hyperactivity detectable in both processes and soma.

\section{(figure 2 close to here)}

\section{Pulse stretching does not reduce photodamage}

For a given fluorophore, microscope and signal, we can reduce photodamage by modifying either the pulse frequency $f$ or duration $\tau$ and adjusting the average laser power $\bar{P}$ accordingly (i.e., keeping either $\bar{P} f=$ const. or $\bar{P} / \tau=$ const., respectively). Longer pulses will reduce the pulse energy but require higher $\bar{P}$. However, whether longer pulses are per se better for biological two-photon imaging has been a matter of debate. The outcome depends on whether photobleaching and photodamage increase more or less rapidly with $\bar{P}$ than the signal. Longer pulses will be neutral if damage processes scale with a power-exponent $m\left(\bar{P}^{m}\right)$ of two, like 2P-excited fluorescence (9) whereas they will be beneficial if higher-order photodamage $(m>2)$ dominates (10). Conversely, longer pulses having a lower peak power and require a higher average power will exacerbate damage processes having a $m<2$, e.g., when one-photon absorbers are present (20) or if tissue heating occurs (21).

We directly compared $\mathrm{Ca}^{2+}$ transients upon 920-nm excitation with 90- (as before) and 172-fs pulses. 90-fs pulses were obtained by a careful optimization of the excitation optical 
path and an optimal pre-chirping in a wavelength- and objective-dependent manner so as to attain shortest pulses in the sample plane (see Methods). 172-fs pulses correspond to the standard 'midline' manufacturer tuning of the DeepSee ${ }^{\mathrm{TM}}$ pulse compressor at $920 \mathrm{~nm}$.

To our surprise, longer pulses and higher laser power ( $\bar{P}$ was adjusted so that astrocytes had an equal GCaMP6f signal for either pulse length, Fig. S4) did not reduce photodamage, Fig. 3A. Aberrant microdomain $\mathrm{Ca}^{2+}$ signals were readily detectable throughout the astrocyte processes, Fig. $3 B$, and somatic activation prevailed, Fig. $3 D$. Quantifying the activity during initial (p1) and terminal 4-min segments (p3) revealed by and large identical results for 172- and 90-fs pulses in the processes (cumulative activity increased $\times 6.0$ vs. $\times 7.5$, p1 vs. p3), Fig. $3 C$. The overall somatic activity in p3 was not significantly different between pulse lengths, Fig. $3 E$, although somatic $\mathrm{Ca}^{2+}$ transients arrived earlier and were of larger amplitude with longer pulses. Our results clearly exclude highly non-linear photodamage as a mechanism for triggering aberrant astrocyte $\mathrm{Ca}^{2+}$ activity.

\section{(figure 3 close to here)}

\section{CW-illumination at $920-\mathrm{nm}$ is as damaging as fs-pulses to the fine processes}

How can we explain that longer pulses and higher peak powers are not more damaging? We hypothesized that contrary to common belief, in the 100-fs and 920-nm excitation regime, photodamage is not dominated by highly non-linear processes like excited-state absorption, absorption from the triplet state, or indirect effects that occur via ROS production through mitochondrial two- and three-photon absorption or lipid oxidation, but rather through direct NIR absorption. This is plausible, because both water and lipid absorption increase by more than one order of magnitude between 800 and $900 \mathrm{~nm}$ (Fig. S5), and so does the risk of focal heating $(m=1)(21,22)$. Thus, different from the short wavelengths $(700-800 \mathrm{~nm})(23)$ that traditionally have been used for $2 \mathrm{P}$ excitation of small chemical indicators, the longer 
wavelengths now commonly used for exciting fluorescent proteins and optogenetic activators might well result in significant absorption and heat production. A simple, testable hypothesis for a linear damage process is that depositing the same average power $\bar{P}$ with a continuouswave (CW) 920-nm laser (i.e., no pulsing) should be equally damaging as pulsed illumination with $2 \mathrm{P}$ fluorescence excitation. This is what we tested next, after having ascertained the effectiveness of our de-modelocking procedure (see Online methods and Fig. S6A, $B$ ).

We designed a "pump-probe" experiment, in which we first imaged cells during 2 min with 90 -fs pulses. We then pursued during $10 \mathrm{~min}$ with either pulsed or continuous-wave (CW) illumination (same $\bar{P}$ ). As no fluorescence is excited with $\mathrm{CW}$ illumination, we read out the resulting $\mathrm{Ca}^{2+}$ signals during another 2-min 'probe' period with pulsed excitation. In a third variant (negative control), we shuttered the laser during $10 \mathrm{~min}$ to allow the cells to recover, Fig. 4A. For each cell, we graphed raster (Fig. 4B) and cumulative-activity plots (Fig. 4C, E). As before (c.f., Fig. 2, Fig. 3), ongoing fs-pulsed illumination led to synchronized 'microdomain $\mathrm{Ca}^{2+}$ activity, Fig. $4 D$ as well as activation of somatic $\mathrm{Ca}^{2+}$ signals, Fig. $4 F$. As hypothesized, CW illumination produced an amount of peripheral $\mathrm{Ca}^{2+}$ hyperactivity that was indistinguishable from pulsed excitation, Figs. $4 D, F$, grey traces. Shuttering the laser between acquisitions virtually abolished this light-induced activity, indicating that the total light dose, not a once triggered irreversible damage cascade produced the aberrant $\mathrm{Ca}^{2+}$ signals, Figs. 4D, F, black traces. Interestingly, and different from the astrocyte processes, $\mathrm{CW}$ illumination did not produce measurable somatic hyperactivity, Fig. $4 F$. Together, our data provide compelling evidence for laser-induced heating being a source of peripheral astrocyte $\mathrm{Ca}^{2+}$ hyperactivity. 


\section{Somatic but not peripheral photodamage is $\mathrm{IP}_{3}$-receptor mediated}

What are possible damage mechanisms in the processes and soma? Perhaps reactive oxygen species (ROS) affect $\mathrm{Ca}^{2+}$ handling by oxidation of the inositol 3-phosphate receptor $\left(\mathrm{IP}_{3} \mathrm{R}\right)$ and sensitization of endoplasmic reticulum (ER) $\mathrm{Ca}^{2+}$ release to promote perisomatic $\mathrm{Ca}^{2+}$ oscillations, mitochondrial $\mathrm{Ca}^{2+}$ uptake (24) and release (25). As the $\mathrm{IP}_{3} \mathrm{R} 2$ is the major, if not only, $\mathrm{IP}_{3} \mathrm{R}$ expressed in astrocytes (26), we took advantage of an $\mathrm{IP}_{3} \mathrm{R} 2$-knock-out (KO) mouse (27) to test if $\mathrm{IP}_{3} \mathrm{R}$ sensitization was involved in mediating somatic damage. As spontaneous $\mathrm{Ca}^{2+}$ signals persist in the processes in $\mathrm{IP}_{3} \mathrm{R} 2-\mathrm{KO}$ mice (28) we speculated if imaging-evoked somatic and peripheral $\mathrm{Ca}^{2+}$ signals could be distinguished through their $\mathrm{IP}_{3} \mathrm{R} 2$-dependence. As before, we ascertained for all experiments equal $\bar{P}$ (Fig. S6C) and mean GCaMP6f-fluorescence at the beginning of the recordings (Fig. S6 $D$ ).

Time-lapse 2P imaging of spontaneous $\mathrm{Ca}^{2+}$ activity GCaMP6f-expressing astrocytes in slices of $\mathrm{IP}_{3} \mathrm{R} 2-\mathrm{KO}$ mice produced heat-induced microdomain $\mathrm{Ca}^{2+}$ transients in the cell periphery as in the WT, Fig. $\mathbf{5 A}$, while the somatic $\mathrm{Ca}^{2+}$ hyperactivity was abolished, Fig. $\mathbf{5} C$. Analysis of initial and terminal 1-min segments respectively, revealed a significant heatinduced $\mathrm{Ca}^{2+}$ activity in the processes, blue on Fig. $5 \mathrm{~B}$, both with respect to the beginning of the same recording and when compared to the second period in wild-type mice when shuttering the laser (data reproduced from Fig. 4D). However, the same robust activity increase in the processes did not lead somatic responses in the $\mathrm{KO}$, as if an $\mathrm{IP}_{3}$-mediated integration of the $\mathrm{Ca}^{2+}$ hyperactivity over different astrocyte branches was required to produce aberrant somatic signals, or ROS production caused by non-linear photodamage causes $\mathrm{IP}_{3^{-}}$

dependent somatic $\mathrm{Ca}^{2+}$ hyperactivity which is mechanistically different from the heattriggered $\mathrm{Ca}^{2+}$ increase in the processes. 


\section{DISCUSSION}

We used state-of-the art methods to image and analyze microdomain $\mathrm{Ca}^{2+}$ signals in cortical astrocytes to show that, (i), 2P-imaging under conditions usually considered safe led to an increase in spontaneous $\mathrm{Ca}^{2+}$ activity; (ii), at constant signal $\left(\bar{P}^{2} / \tau=\right.$ const. $)$, stretched pulses and increased average power failed to reduce photodamage, thus excluding non-linear damage mechanisms; (iii) 920-nm CW excitation evoked aberrant microdomain $\mathrm{Ca}^{2+}$ signals in the astrocyte processes with a comparable efficiency than pulsed excitation, confirming a onephoton absorption mechanism; $(v)$, genetic ablation of the major astroglial $\mathrm{IP}_{3}$ receptor abolished somatic $\mathrm{Ca}^{2+}$ transients, but it did not affect heat-mediated damage in the fine processes, pointing to different damage mechanisms in the cell body and periphery.

\section{Brain heating as a damage mechanism}

Heating was discussed as a possible damage mechanism in non-linear microscopies before the advent of the $2 \mathrm{P}$ microscope (29). Sheppard predicted permissive $\bar{P}$ delivered to a single excitation spot around $35 \mathrm{~mW}$ and $100 \mathrm{~mW}$ for stationary illumination and fast scanning, respectively, which seems optimistic in view of reported biological damage thresholds closer to $10 \mathrm{~mW}$ (9-11). Comparing fs vs. ps pulses Schönle and Hell judged focal heating irrelevant at $750-800 \mathrm{~nm}$ (the dominant spectral window for exciting small-molecule chemical indicators), but neither they used a biological readout, nor is it clear if the biology under study would really tolerate the predicted $0.5-3^{\circ} \mathrm{C}$-change (23). Morphological damage of unlabeled cultured cells exposed to fs and ps pulses $(780 \mathrm{~nm}, 60 \mu \mathrm{s})$ followed an approximate $\bar{P}^{2} / \tau$ law, suggesting a damage exponent close to $m=2$ (20), but more subtle forms of photodamage than visible destruction were not assessed in this study. For slightly longer wavelengths, Marcias-Romero et al. estimated a $1-5^{\circ} \mathrm{C}$ increase in the focus of a $1035-\mathrm{nm}$ laser beam $(80 \mathrm{MHz}, 1.1 \mathrm{NA})$ in water for typical pixel dwell times $<10 \mu \mathrm{s}(30)$ and argued 
for wide-field illumination and low-repetition rate $2 \mathrm{P}$ imaging to reduce thermal damage. Using the temperature-dependent Stokes shift of Laurdan, a $1{ }^{\circ} \mathrm{C} / 100 \mathrm{~mW}$ change was reported for a CW 1064-nm trapping beam in the 20- to 200-mW regime (31). Along the same lines, Schmidt and co-workers predicted a temperature rise around $5^{\circ} \mathrm{C} / 100 \mathrm{~mW}$ for various trapping beams based on a model taking into account light absorption in the neighborhood of the focus, outward heat flow and heat sinking by the glass surfaces of the sample chamber (32). A temperature increase of the same order of magnitude $\left(1.8^{\circ} \mathrm{C} / 100\right.$ $\mathrm{mW}$ ) was measured with thermocouple probes and quantum-dot nanothermometers upon $2 \mathrm{P}$ single-spot scanning in the neocortex of awake or anesthetized mice, in vivo, and $\bar{P}>250$ $\mathrm{mW}$ produced irreversible thermal damage (21).

Our results underpin the importance of thermal damage for two-photon excitation in the biologically important spectral window around $920 \mathrm{~nm}$ and call for a necessary degree of caution when designing and interpreting experiments using 2P-brain imaging and photostimulation. Wavelengths $>900 \mathrm{~nm}$ are now commonly being used for the excitation of fluorescent proteins, genetically encoded $\mathrm{Ca}^{2+}$ indicators (GECIs) and for the optogenetic activation of opsin-expressing cells. NIR light at these wavelengths is more invasive than often thought, particularly when imaging at high spatio-temporal resolution or when using multiple-spot schemes that increase the power deposit in tissue.

As thermal effects rely on the accumulation and dissipation of energy, they depend on $\bar{P}$, but also on parameters like the focal spot size, scanning parameters and the pulse frequency and shape. Moreover, the heat deposit will be additive in multi-spot, line-scanning, and holographic illumination schemes, and scanning with high spatial and temporal frequencies will intensify thermal damage. On the other hand, external factors like brain cooling due to a cranial window (33) or perfusion with temperature-controlled ASCF can contribute to equilibrating temperature gradients. 
At a fixed pulse length, we expect lower pulse repetition rates, shorter pixel dwell times and re-scanning (i.e., temporal averaging on a $\mu$ s time-scale) (34) to be beneficial. In view of thermal diffusion rates of $\sim 1 \mu \mathrm{m}^{2} / \mu \mathrm{s}$, scanning schemes in which neighboring pixels are excited in a spatially interspersed manner rather than sequentially will favor the dissipation of locally generated heat. A low overall duty cycle (i.e., recording from multiple, distant and small ROIs rather than from full frames, temporally spacing short bursts of images taken at high frequency (21)) should be beneficial to mitigate build-up of thermal damage. Random-access scanning (35-37) will be advantageous for applications where high spatiotemporal sampling is indispensible like imaging dendritic spines, astrocyte processes, or for 2P-STED microscopy.

Our work alerts the biological microscopist to the importance of other damage mechanisms that coexist with localized non-linear photodamage $(10,38)$ and it also calls for prudence in the ongoing quest for ever-longer wavelength IR-excited and red-shifted probes for deep tissue imaging, because water and lipid absorption $\left(\mu_{\mathrm{a}}\right)$ continue to increase. However, tissue scattering $\left(\mu_{\mathrm{s}}\right)$ is reduced at these wavelengths. Thus, the choice of the optimal fluorophore and excitation wavelength windows will be a trade-off between absorption and tissue scattering $(39,40)$. Finally, thermal damage is a particular concern for deep-tissue imaging because compensating for the exponential excitation losses with increasing depth will expose the tissue surface to very high laser powers that - even unfocused - will be harmful due to surface heat generation.

\section{Somatic $\mathrm{Ca}^{2+}$ signals are a sensitive readout for damage}

Although somatic $\mathrm{Ca}^{2+}$ transients have been used as a proxy for astrocyte activity, $\geq 90 \%$ of spontaneous $\mathrm{Ca}^{2+}$ activity occurs in the cell periphery $(14,17,41-43)$. We confirm this observation (Figs.1,2) and find that astrocyte somata are mostly silent under conditions of 
2P-images that preserve physiological $\mathrm{Ca}^{2+}$ signaling. Even when neuronal action potentials were not blocked with TTX, somatic $\mathrm{Ca}^{2+}$ signals were sparse, making their appearance a sensitive and facile readout for damage, however, heat-induced alterations of microdomain $\mathrm{Ca}^{2+}$ activity in the cell periphery occurred earlier (Fig.2) and were more difficult to spot.

Light-induced somatic $\mathrm{Ca}^{2+}$ transients in cortical astrocytes depend on $\mathrm{IP}_{3}$-mediated mechanisms (Fig.5). In the cell periphery with its large membrane-to-cytosol ratio, other mechanisms prevail. Candidates are channels with thermosensitive gating in the physiological temperature range, like TRPV3 (44), STIM-1 (45), and a number of voltage-gated channels (46). Temperature variations around $37^{\circ} \mathrm{C}$ also sensitize TRPV4 channels that are expressed in roughly $1 / 3$ of astrocytes (47) to diverse stimuli triggering channel opening (48). A different $\mathrm{Ca}^{2+}$-influx pathway potentially recruited following NIR illumination and regulated by oxidative stress is TRPM2 (49). Alternatively, heat-induced pore-formation or lipid rearrangements in the membranes of $\mathrm{Ca}^{2+}$ stores or the plasma membrane could mediate microdomain $\mathrm{Ca}^{2+}$ hyperactivity.

\section{Towards even shorter pulses?}

Our study illustrates the potential benefit of shorter pulses and lower $\bar{P}$ for $2 \mathrm{P}$-excitation of GCaMP-based indicators at $920 \mathrm{~nm}$ and in the 100-fs regime. 90 -fs were the minimum we could attain with our laser, microscope, objective and pre-compensation optics, but even shorter pulses might be beneficial $(50,51)$. This strategy will eventually be bounded by some shortest tolerable pulse length because the resulting high peak powers will favor non-linear photo-bleaching and -damage pathways . The demonstration that phase-optimized pulses with 28 -fs length reduced the bleaching rate of EGFP by a factor of 4 while maintaining the same intensity of the fluorescence signal (52) let us predict that there might be room for a further reduction of NIR-induced damage for brain imaging with even shorter pulses. 


\section{METHODS}

Full experimental procedures are available online.

\section{ACKNOWLEDGEMENTS}

We thank Cendra Agulhon (Paris) for providing the $\mathrm{IP}_{3} \mathrm{R} 2-\mathrm{KO}$ mouse line and immunofluorescence data, Frank Pfrieger (Strasbourg) for providing the GLASTcreER ${ }^{\mathrm{T} 2}$ mouse line, Marcel van 't Hoff for programming the pulse-length adjustment in LABVIEW and Stéphane Dieudonné (Paris), Manfred Lindau (Göttingen/Cornell) and many other colleagues for helpful discussions. Patrice Jegouzo, Christophe Tourain (workshop) and the animal house team provided excellent technical assistance.

Financed by the European Union (FP6-STRP “AUTO-SCREEN”, FP7 ERA-NET NEURON "NANOSYN", FP7 JPND "SYNSPREAD”, H2020 EUROSTARS “OASIS"), the FranceBioImaging large-scale national infrastructure initiative (FBI, ANR-10-INSB-04, Investments for the future) and the Region Ile de France (Cancéropôle, "EDISON"). The Oheim lab is a member of the Ecole de Neurosciences de Paris (ENP) and the C'nano Ile-deFrance excellence clusters for neurobiology and nanobiophotonics, respectively.

This work contains supporting online material. 


\section{Figure legends}

Fig. 1. Properties of spontaneous astrocyte $\mathbf{C a}^{2+}$ signals. (A) Diffraction-limited 920-nm excited two-photon (2P) fluorescence image across a single equatorial plane of a GCaMP6fexpressing astrocyte in an acute slice of the murine barrel cortex. Imaging depth $83 \mu \mathrm{m}$. Top, morphological overview from the time-average of 120 consecutive frames. Bottom, regions of interest (ROIs) corresponding to the detected $\mathrm{Ca}^{2+}$-transients (color for better discrimination). Overlapping ROIs represent activity occurring in the same area at different time points. Red central ROI is the soma. Scale bar, $10 \mu \mathrm{m}$. (See Movie S1). (B) Black, area-scaled fluorescence traces of spontaneous $\mathrm{Ca}^{2+}$ events $\left(\Delta F / F_{0}\right.$ multiplied by the area of the corresponding ROI) ordered, from top to bottom, according to their distance to soma. See Fig. S2 and Online Methods for details on the detection algorithm. Bottom, grey, summed activity over all events in the astrocyte processes. Red trace somatic activity, the soma was silent throughout the recording.

\section{Fig. 2. 2P excitation at $920 \mathrm{~nm}$ triggers a subtle but detectable $\mathrm{Ca}^{2+}$ hyperactivity. $(A)$}

Black, raster plot of the dat in Fig. $1 B$, along with its further evlution during a recording lasting more than $16 \mathrm{~min}$ (500 frames, $0.5 \mathrm{~Hz}$ ). Grey, increase of the summed activity over all processes. Red, the initially silent soma becomes progressively active. (B) Summed peripheral (grey) and somatic (red) activities under the same conditions as in (A) for $n=8$ cells in $N=6$ mice. Mean activity was quantified during periods p1: 0-240 s, p2: 240-480 s, p3: 760-1000 s, boxed. (C) Pseudo-color overlay of the events detected during p1, p2 and p3 for the cell in (A). $\mathrm{Ca}^{2+}$-activity increasingly occurs at similar spatial locations during $\mathrm{p} 2$ and $\mathrm{p} 3$. Scale bar, 10 $\mu \mathrm{m}$. (D) Parameters characterizing $\mathrm{Ca}^{2+}$ transients during $\mathrm{p} 1, \mathrm{p} 2$ and $\mathrm{p} 3$. Bar plots show population median \pm SEM, symbols graph individual cells. Event frequency increases from $\mathrm{p} 1$ to $\mathrm{p} 2$ and between $\mathrm{p} 1$ and p3 (two-sided nonparametric Wilcoxon-Mann-Whitney two-sample rank test). Light-induced $\mathrm{Ca}^{2+}$ signals resemble spontaneous $\mathrm{Ca}^{2+}$ microdomains, which makes them difficult to distinguish. (E) Grey, comparison of the summed activity in the astrocyte processes, i.e., integral of the traces shown in (B), reveals a net increase between p1 vs. p2 
and p1 vs. p3. Red, the somatic activity, corresponding to the red traces in (B), remains significantly unchanged during all recording periods, although a trend to progressively higher activity is seen. $*: P<0.05, * *: P<0.01, * * *: P<0.005$, n.s.: not significant.

\section{Fig. 3. Longer, less energetic pulses do not reduce light-evoked $\mathrm{Ca}^{2+}$-hyperactivity. $(A)$} Right, raster plot of 920-nm light-induced $\mathrm{Ca}^{2+}$ hyperactivity as in Fig. $2 \mathrm{~A}$, but for a different cell. Pulses were carefully optimized as in the both previous figures to achieve the shortest possible pulses under the objective (see Online methods), resulting in 90 -fs pulse length $\left(\operatorname{sech}^{2}\right)$. See Movie S2 for a recording of this cell. Left, same for a cell excited with 172-fs pulses, i.e., with lower peak power but higher average laser power to maintain a constant initial signal, i.e., $\bar{P}^{2} / \tau=$ const. $(B)$ Red, summed activity as in Fig. $2 B$ ( $n=8$ cells/6 mice at $90 \mathrm{fs}$ ) and side-to-side comparison with summed activity upon 172-fs excitation (blue). (C) $\mathrm{Ca}^{2+}$ activities in $\mathrm{p} 3$ compared to $\mathrm{p} 1$ (defined as in Fig. $2 B$ ) increase (median \pm SEM) for both 90-fs (red, same data as in Fig. 2E) and 172-fs pulses (blue, $n=7$ cells/6 mice). Furthermore, activity was undistinguishable between short and long pulses for $\mathrm{p} 1$ (validating our experimental paradigm) but also during p3, i.e., contrary to what was expected for a highly non-linear photodamage no significant difference was found for different pulse energies at (one-sided non-parametric Wilcoxon-Mann-Whitney two-sample rank test). (D) Somatic $\Delta F / F_{0}$ traces for 90 -fs ( $r e d$, corresponding to Fig. $2 B$ ) and 172-fs pulses (blue). (E), Statistical analysis showed that the somatic activity was indistinguishable between 90-fs (red, corresponding to Fig. 2 E) and 172-fs pulses (blue), both during p1 and p3. *: $P<0.05$, **: $P$ $<0.01, * * *: P<0.005$, n.s.: not significant.

\section{Fig. 4. Light-induced $\mathrm{Ca}^{2+}$ hyperactivity depends on total light dose, not the pulse energy.}

(A) Schematic representation of the experiment design. Spontaneous $\mathrm{Ca}^{2+}$-transients were 
recorded during a 2-min control period (p1; $920 \mathrm{~nm}, 90 \mathrm{fs}, 0.5 \mathrm{~Hz})$. Then, during 10 minutes, three different protocols were applied: $(i)$, the cells were either imaged as in the preceding figures (i.e., same scenario as before) or, (ii), the laser was shuttered and the cells allowed to recover from the previous recording. In a $3^{\text {rd }}$ variant, (iii), we de-modelocked the laser to expose the cells to continuous-wave (CW) radiation with the same average power $\bar{P}$ as during pulsed excitation (see Online Methods and Fig. S6). The respective impact of these different protocols on the astrocytic $\mathrm{Ca}^{2+}$ activity was read out in another 2-min recording (same parameters as $\mathrm{p} 1$ ). (B) Raster plots illustrate the outcome of the three different protocols. (C) Cumulative $\mathrm{Ca}^{2+}$ activity traces in the astrocyte processes during $\mathrm{p} 1$ and $\mathrm{p} 3$. (D) Statistical analysis of data shown in (C). Activity increases for all conditions between the beginning of p1 (0-60s) and end of p3 (660-720 s; one-sided non-parametric Wilcoxon-MannWhitney two-sample rank test). Of note, the $\mathrm{Ca}^{2+}$-activity in the processes during $\mathrm{p} 3$ is indistinguishable for exposure to pulsed vs. CW light, both of which are significantly higher than with the laser shuttered. All experiments started from initial activity levels during p1. (n.s. not shown for clarity). (E), Somatic $\Delta F / F_{0}$ traces for the three scenarios and statistical analysis in (F). For CW illumination, somatic activity is significantly higher in p3 compared to either no or pulsed excitation during $\mathrm{p} 2$, respectively. $*: P<0.05$, **: $P<0.01$, ***: $P<$ 0.005, n.s.: not significant.

Fig. 5. Somatic but not peripheral $\mathrm{Ca}^{2+}$-hyperactivity is $I P_{3} R 2$ receptor dependent. (A) Cumulative $\mathrm{Ca}^{2+}$ activity in the astrocyte processes during a 12-min recording $(920 \mathrm{~nm}, 90 \mathrm{fs}$, $0.5 \mathrm{~Hz}$ ) of GCaMP6f-expressing astrocytes from $\mathrm{IP}_{3} \mathrm{R} 2 \mathrm{KO}$ mice shows a pronounced lightinduced $\mathrm{Ca}^{2+}$-hyperactivity in the processes ( $n=3$ cells from 2 mice). (B) Comparison of cumulative activity in the processes of $\mathrm{IP}_{3} \mathrm{R} 2 \mathrm{KO}$ mice during beginning of p1 (0-60s) and end of p3 (660-720s) (p1 and p3 same as in in Fig. 4, light blue graphs, one-sided nonparametric Wilcoxon-Mann-Whitney two-sample rank test). No significant difference is observed for either $\mathrm{p} 1$ or $\mathrm{p} 3$ between $\mathrm{IP}_{3} \mathrm{R} 2 \mathrm{KO}$ or wild-type (WT) mice (data from Fig. 4D), 
whereas the $\mathrm{KO}$ data during $\mathrm{p} 3$ is significantly different from the same period when the laser was shuttered (data from Fig. 4D), confirming that peripheral $\mathrm{Ca}^{2+}$ hyperactivity persisted in the absence of the $\mathrm{IP}_{3}$-mediated signaling pathways. In contrast, $(\mathrm{C})$, Soma of $\mathrm{IP}_{3} \mathrm{R} 2 \mathrm{KO}$ astrocytes are silent during the whole recording period in all cells. (D) In fact, somatic activity in $\mathrm{IP}_{3} \mathrm{R} 2 \mathrm{KO}$ mice is more similar to the WT astrocytes that were only imaged during $\mathrm{p} 1$ and p3 with the laser shuttered in between than to those that were exposed to continuous $2 \mathrm{PEF}$ imaging (c.f., Fig. $4 F$ ). *: $P<0.05$, **: $P<0.01$, ***: $P<0.005$, n.s.: not significant. 


\section{References}

1. Denk W, Strickler JH, \& Webb WW (1990) Two-photon laser scanning fluorescence microscopy. Science 248(4951):73-76.

2. Stosiek C, Garaschuk O, Holthoff K, \& Konnerth A (2003) In vivo two-photon calcium imaging of neuronal networks. Proc. Natl. Acad. Sci. USA 100(12):7319-7324.

3. Helmchen F \& Denk W (2005) Deep tissue two-photon microscopy. Nat. Methods 2(12):932-940.

4. Svoboda K \& Yasuda R (2006) Principles of two-photon excitation microscopy and its applications to neuroscience. Neuron 50(6):823-839.

5. Mao T, O'Connor DH, Scheuss V, Nakai J, \& Svoboda K (2008) Characterization and subcellular targeting of GCaMP-type genetically-encoded calcium indicators. PloS One 3(3):e1796.

6. Looger LL \& Griesbeck O (2012) Genetically encoded neural activity indicators. Curr. Op. Neurobiol. 22(1):18-23.

7. Akerboom J, et al. (2013) Genetically encoded calcium indicators for multi-color neural activity imaging and combination with optogenetics. Front. Mol. Neurosci. 6.

8. Patterson GH \& Piston DW (2000) Photobleaching in two-photon excitation microscopy. Biophys. J. 78(4):2159-2162.

9. Koester HJ, Baur D, Uhl R, \& Hell SW (1999) $\mathrm{Ca}^{2+}$ fluorescence imaging with picoand femtosecond two-photon excitation: signal and photodamage. Biophys. $J$. 77(4):2226-2236.

10. Hopt A \& Neher E (2001) Highly nonlinear photodamage in two-photon fluorescence microscopy. Biophys. J. 80(4):2029-2036.

11. Tan Y, Llano I, Hopt A, Würriehausen F, \& Neher E (1999) Fast scanning and efficient photodetection in a simple two-photon microscope. J. Neurosci. Meth. 92(1):123-135.

12. Weisenburger S, Prevedel R, \& Vaziri A (2017) Quantitative evaluation of twophoton calcium imaging modalities for high-speed volumetric calcium imaging in scattering brain tissue. bioRxiv:115659.

13. Ohkura M, et al. (2012) Genetically encoded green fluorescent $\mathrm{Ca}^{2+}$ indicators with improved detectability for neuronal Ca2+ signals. PloS one 7(12):e51286.

14. Shigetomi E, Kracun S, Sofroniew MV, \& Khakh BS (2010) A genetically targeted optical sensor to monitor calcium signals in astrocyte processes. Nat. Neurosci. 13(6):759-766.

15. Haustein MD, et al. (2014) Conditions and constraints for astrocyte calcium signaling in the hippocampal mossy fiber pathway. Neuron 82(2):413-429.

16. Rungta RL, et al. (2016) $\mathrm{Ca}^{2+}$ transients in astrocyte fine processes occur via $\mathrm{Ca} 2+$ influx in the adult mouse hippocampus. Glia 64(12):2093-2103.

17. Bindocci E, et al. (2017) Three-dimensional $\mathrm{Ca}^{2+}$ imaging advances understanding of astrocyte biology. Science 356(6339):eaai8185.

18. Tian L, et al. (2009) Imaging neural activity in worms, flies and mice with improved GCaMP calcium indicators. Nature Meth 6(12):875-881.

19. Ye L, Haroon MA, Salinas A, \& Paukert M (2017) Comparison of GCaMP3 and GCaMP6f for studying astrocyte $\mathrm{Ca}^{2+}$ dynamics in the awake mouse brain. PloS One 12(7):e0181113.

20. König K, Becker TW, I. F, Riemann I, \& Halbhuber KJ (1999) Pulse-length dependence of cellular response to intense near-infrared pulses in multiphoton microscopes. Opt. Lett. 24:113-115. 
21. Podgorski K \& Ranganathan G (2016) Brain heating induced by near-infrared lasers during multiphoton microscopy. J. Neurophysiol. 116:1012-1023.

22. Debarre D, Olivier N, Supatto W, \& Beaurepaire E (2014) Mitigating phototoxicity during multiphoton microscopy of live Drosophila embryos in the $1.0-1.2 \mu \mathrm{m}$ wavelength range. PLoS One 9(8):e104250.

23. Schönle A \& Hell SW (1998) Heating by absorption in the focus of an objective lens. Opt. Lett. 23(5):325-327.

24. Bánsághi S, et al. (2014) Isoform-and species-specific control of inositol 1, 4, 5trisphosphate $\left(\mathrm{IP}_{3}\right)$ receptors by reactive oxygen species. J. Biol. Chem. 289(12):81708181 .

25. Agarwal A, et al. (2017) Transient opening of the mitochondrial permeability transition pore induces microdomain calcium transients in astrocyte processes. Neuron 93(3):587-605. e587.

26. Sherwood MW, et al. (2017) Astrocytic IP3Rs: Contribution to $\mathrm{Ca}^{2+}$ signalling and hippocampal LTP. Glia 65(3):502-513.

27. Petravicz J, Boyt KM, \& McCarthy KD (2014) Astrocyte $\mathrm{IP}_{3} \mathrm{R} 2$-dependent $\mathrm{Ca}^{2+}$ signaling is not a major modulator of neuronal pathways governing behavior. Front. Behav. Neurosci. 8:384.

28. Srinivasan $\mathrm{R}$, et al. (2015) $\mathrm{Ca} 2+$ signaling in astrocytes from $\mathrm{IP}_{3} \mathrm{R} 2^{-/-}$mice in brain slices and during startle responses in vivo. Nat. Neurosci. 18(5):708-717.

29. Sheppard CJR \& Komphner R (1978) Resonant scanning optical microscope. Appl. Opt. 17(18):2879-2881.

30. Macias-Romero C, Zubkovs V, Wang S, \& Roke S (2016) Wide-field mediumrepetition-rate multiphoton microscopyreduces photodamage of living cells. Biomed. Opt. Express 7(4):1458-1467.

31. Liu Y, Sonek G, Berns M, \& Tromberg B (1996) Physiological monitoring of optically trapped cells: assessing the effects of confinement by 1064-nm laser tweezers using microfluorometry. Biophys. J. 71(4):2158-2167.

32. Peterman EJ, Gittes F, \& Schmidt CF (2003) Laser-induced heating in optical traps. Biophys. J. 84(2):1308-1316.

33. Kalmbach AS \& Waters J (2012) Brain surface temperature under a craniotomy. $J$. Neurophysiol. 108(11):3138-3146.

34. Chen X, et al. (2012) LOTOS-based two-photon calcium imaging of dendritic spines in vivo. Nat. Prot. 7(10):1818.

35. Iyer V, Hoogland TM, \& Saggau P (2006) Fast functional imaging of single neurons using random-access multiphoton (RAMP) microscopy. J. Neurophysiol. 95(1):535545.

36. Salome R, et al. (2006) Ultrafast random-access scanning in two-photon microscopy using acousto-optic deflectors. J. Neurosci. Meth. 154(1-2):161-174.

37. Reddy GD, Kelleher K, Fink R, \& Saggau P (2008) Three-dimensional random access multiphoton microscopy for functional imaging of neuronal activity. Nat. Neurosci. 11(6):713-720.

38. Icha J, Weber M, Waters JC, \& Norden C (2017) Phototoxicity in live fluorescence microscopy, and how to avoid it. Bioessays 39(8).

39. Horton NG, et al. (2013) In vivo three-photon microscopy of subcortical structures within an intact mouse brain. Nat. Photon. 7(3):205.

40. Guesmi K, et al. (2018) Dual-color deep-tissue three-photon microscopy with a multiband infrared laser. Light: Sci. Appl. in press. 
41. Nett WJ, Oloff SH, \& Mccarthy KD (2002) Hippocampal astrocytes in situ exhibit calcium oscillations that occur independent of neuronal activity. J. Neurophysiol. 87(1):528-537.

42. Wang X, et al. (2006) Astrocytic $\mathrm{Ca}^{2+}$ signaling evoked by sensory stimulation in vivo. Nat. Neurosci. 9(6):816.

43. Sun M-Y, et al. (2014) Astrocyte calcium microdomains are inhibited by Bafilomycin A1 and cannot be replicated by low-level Schaffer collateral stimulation in situ. Cell Calcium 55(1):1-16.

44. Xu H, et al. (2002) TRPV3 is a calcium-permeable temperature-sensitive cation channel. Nature 418(6894):181.

45. Xiao B, Coste B, Mathur J, \& Patapoutian A (2011) Temperature-dependent STIM1 activation induces $\mathrm{Ca} 2+$ influx and modulates gene expression. Nat. Chem. Biol. 7(6):351-358.

46. Chowdhury S, Jarecki BW, \& Chanda B (2014) A molecular framework for temperature-dependent gating of ion channels. Cell 158(5):1148-1158.

47. Shibasaki K, Ikenaka K, Tamalu F, Tominaga M, \& Ishizaki Y (2014) A novel subtype of astrocytes expressing TRPV4 (transient receptor potential vanilloid 4) regulates neuronal excitability via release of gliotransmitters. J. Cell Biol. 289(21):14470-14480.

48. Gao X, Wu L, \& O'Neil RG (2003) Temperature-modulated diversity of TRPV4 channel gating activation by physical stresses and phorbol ester derivatives through protein kinase C-dependent and-independent pathways. J. Biol. Chem. 278(29):2712927137.

49. Kühn FJ, Heiner I, \& Lückhoff A (2005) TRPM2: a calcium influx pathway regulated by oxidative stress and the novel second messenger ADP-ribose. Pflügers Arch. 451(1):212-219.

50. Xi P, Andegeko Y, Weisel LR, Lozovoy VV, \& Dantus M (2008) Greater signal, increased depth, and less photobleaching in two-photon microscopy with $10 \mathrm{fs}$ pulses. Opt. Commun. 281(7):1841-1849.

51. Pang S, Yeh AT, Wang C, \& Meissner KE (2009) Beyond the 1/Tp limit: two-photonexcited fluorescence using pulses as short as sub-10-fs. J. Biomd. Opt. 14(5):054041.

52. Kawano H, et al. (2003) Attenuation of photobleaching in two-photon excitation fluorescence from green fluorescent protein with shaped excitation pulses. Biochem. Biophys. Res. Commun. 311(3):592-596. 


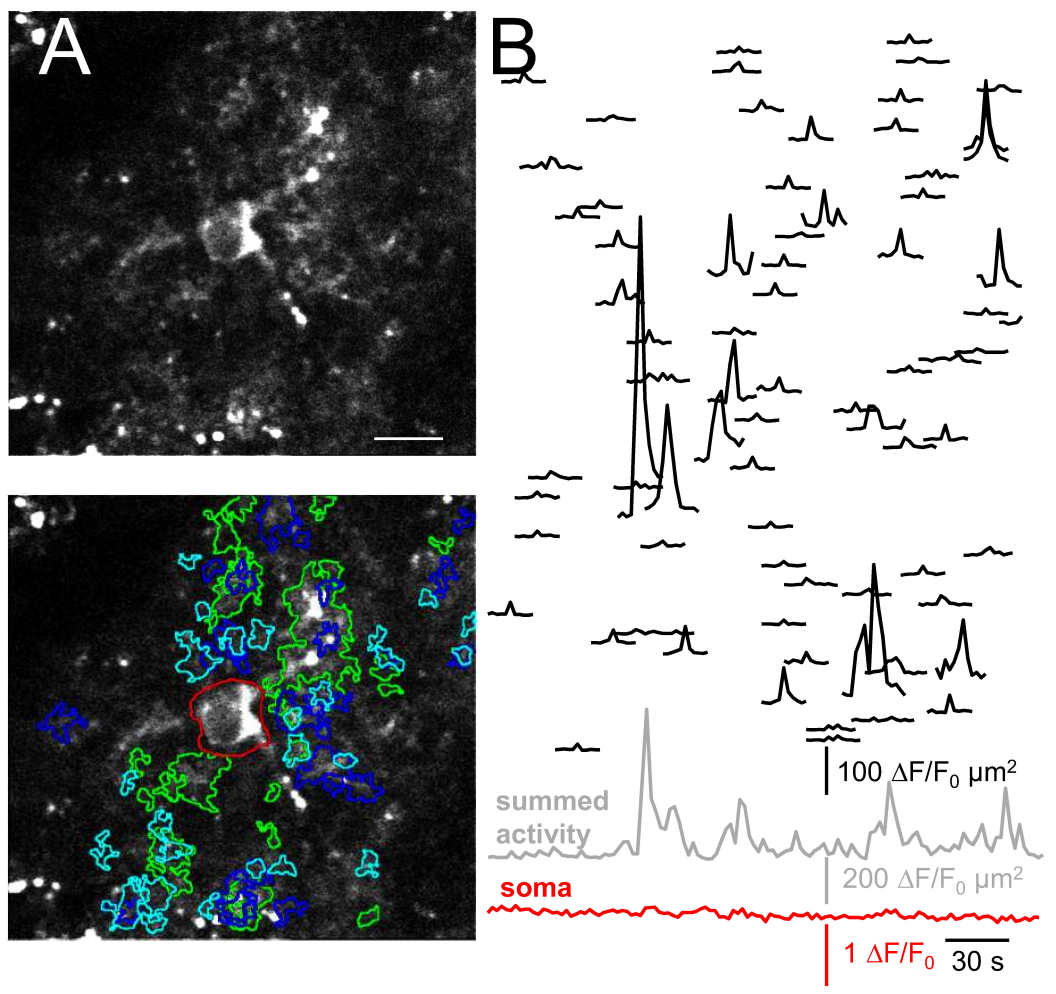


summed

activity

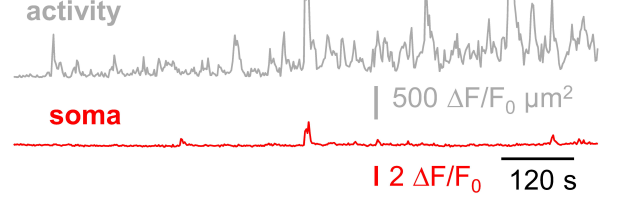

I $2 \Delta \mathrm{F} / \mathrm{F}_{0} \overline{120 \mathrm{~s}}$

ROI number

( $\mathrm{n}=8$ cells)

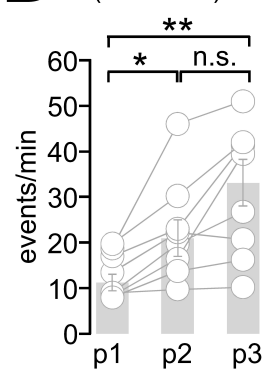

ROI area

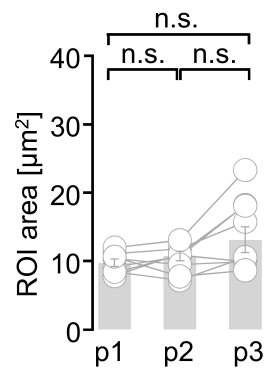

B Processes optimized pulses 90 fs $(n=8$ cells)

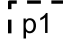

I

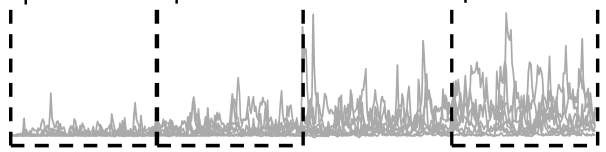
Soma

|500 $\Delta \mathrm{F} / \mathrm{F}_{0} \mu \mathrm{m}^{2}$

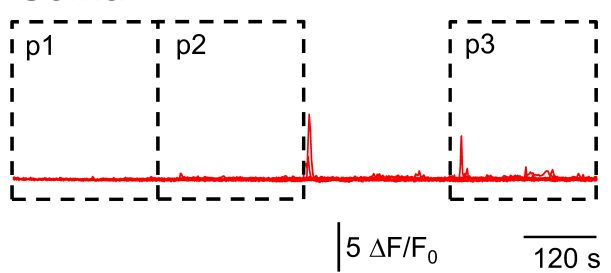

Peak height

Peak duration

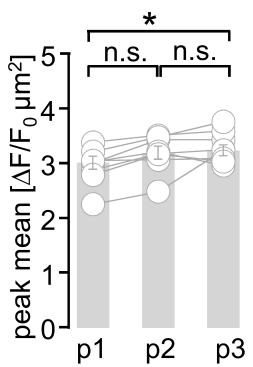

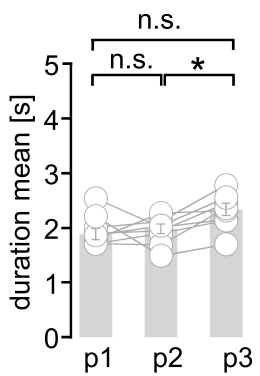

0

․ㅜㅇ

స̃

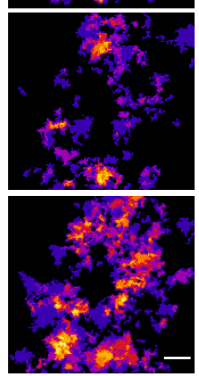

7 ROls

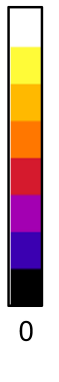

Soma Integral

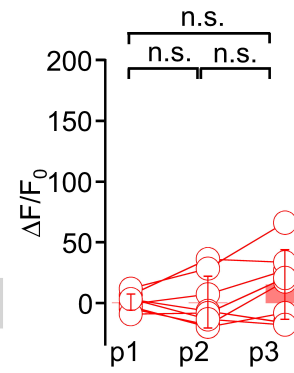


A optimized pulses 90 fs

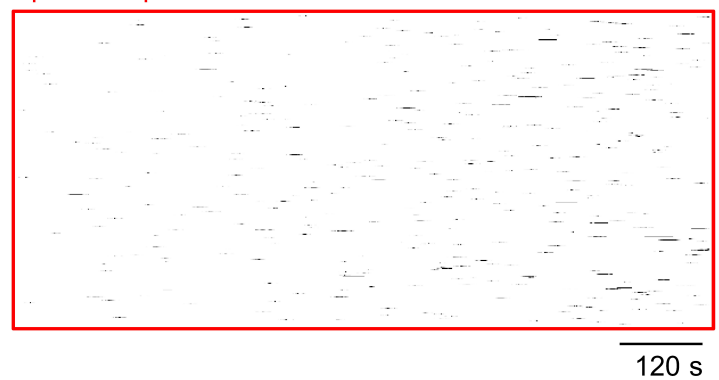

B Processes

optimized pulses $90 \mathrm{fs}$ ( $n=8$ cells)
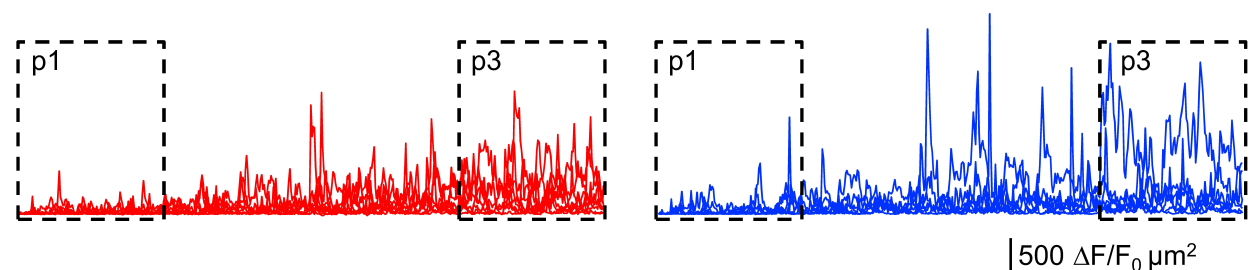

longer pulses 172 fs

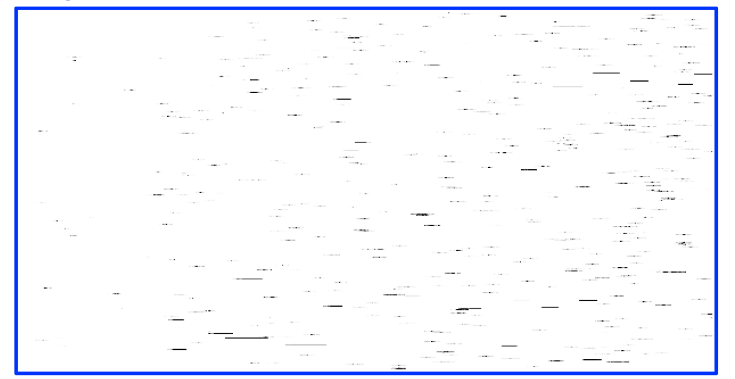

\section{DSoma}

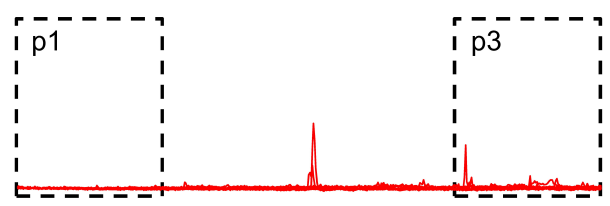

longer pulses 172 fs ( $n=7$ cells)

C

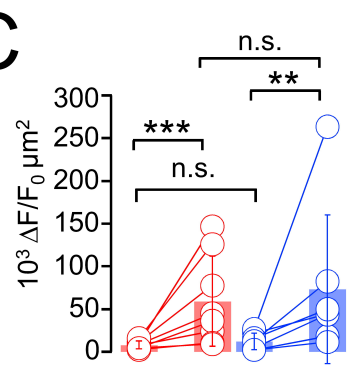

$E$

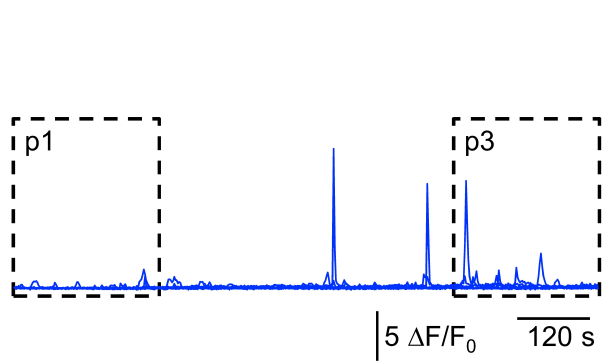


$A$
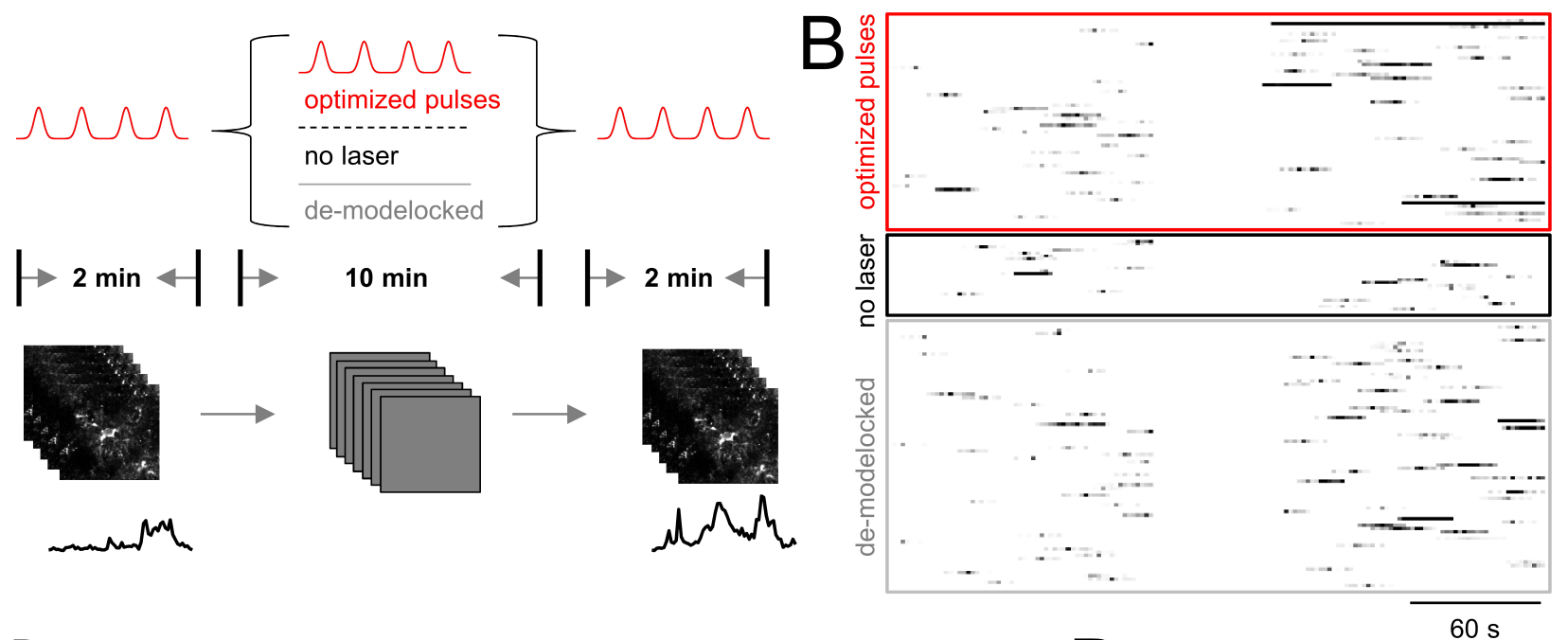

\section{Processes}
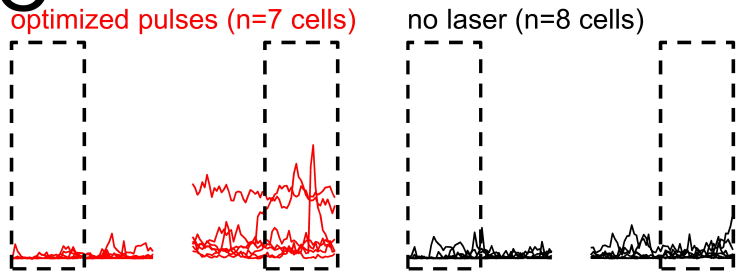

\section{E soma}
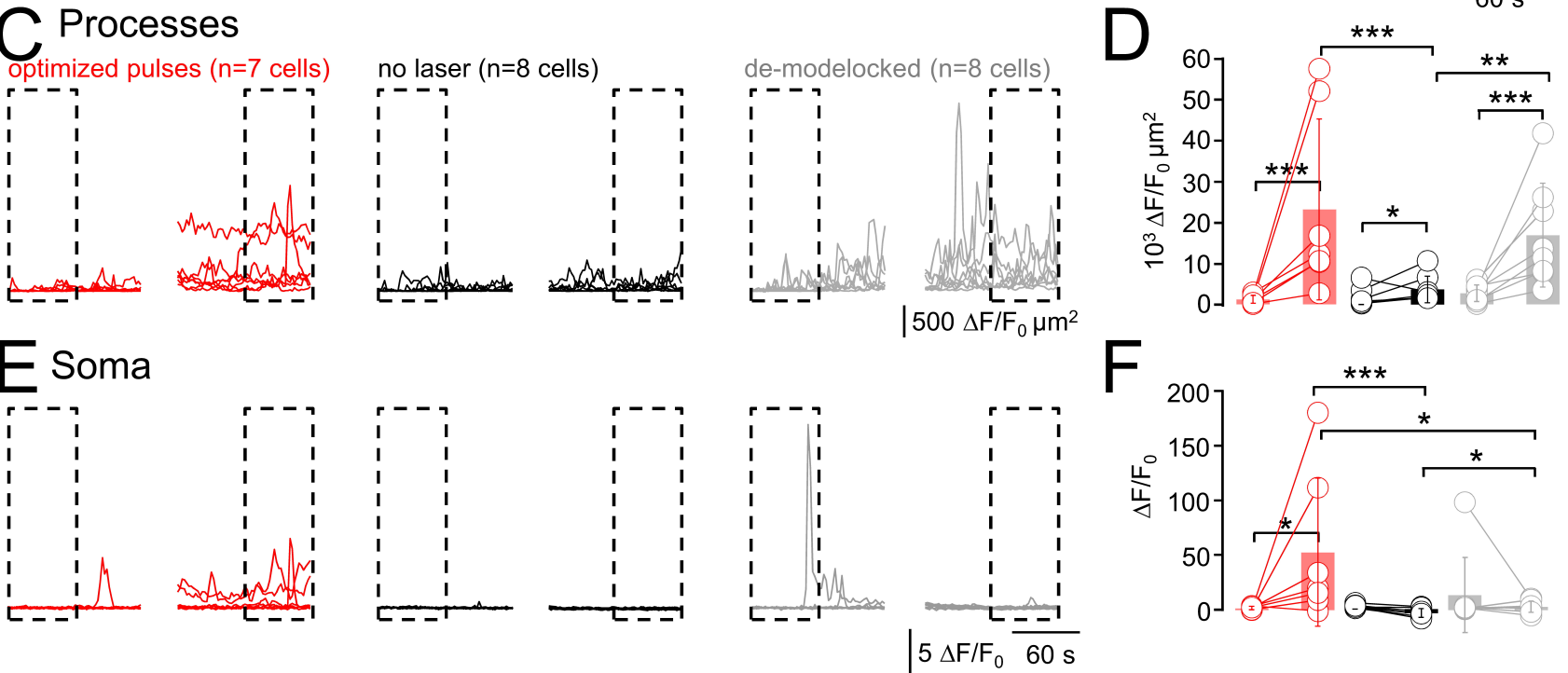

$5 \Delta \mathrm{F} / \mathrm{F}_{0} \frac{--1}{60 \mathrm{~s}}$ 


\section{A Processes}

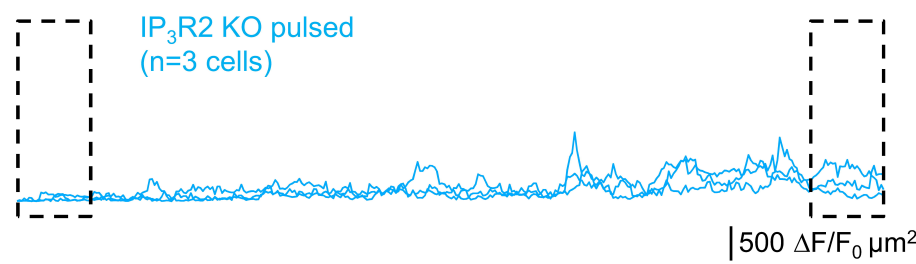

\section{Soma}

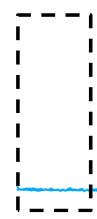

B

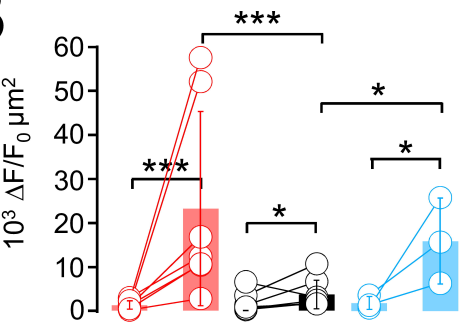

D

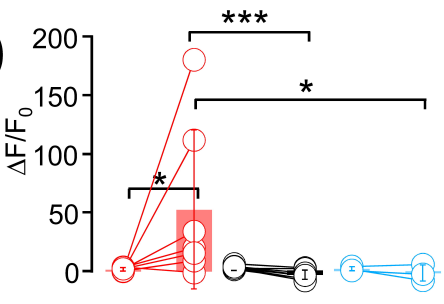

$I_{3}$ R2 WT IP ${ }_{3}$ R2 WT IP IP $_{3}$ R KO pulsed

$\mathrm{n}=7$ no lase $\mathrm{n}=8$ $n=3$ 